\title{
Synthesis and optical properties of conjugated polymers bearing a 1,8-difunctionalized carbazole unit
}

\author{
Koji Takagi, Hidenobu Takao and Tsuyoshi Nakagawa
}

Three conjugated polymers P1-P3 bearing the 1,8-difunctionalized/9-unsubstituted carbazole unit were synthesized by the Suzuki coupling polymerization technique using 9,9-dihexylfluorene-2,7-diboronic acid as the co-monomer. The functional groups at the 1,8-positions were imine (P1), 1,3-oxazoline (P2) and pyridine (P3). The conjugated polymers bearing 1,8-dipyridyl-9-methylcarbazole (P4) and 1,8-diphenylcarbazole (P0) were also prepared. Although the imine $\mathrm{C}=\mathrm{N}$ bond in 3,6-dibromo-1,8-bis((phenylimino)methyl)carbazole was partially hydrolyzed under the polymerization condition, the carbazole monomer that had the sterically demanding imine group successfully gave the defect-free conjugated polymer P1. From the ${ }^{1} \mathrm{H}-\mathrm{NMR}$ (nuclear magnetic resonance) and infrared spectra, the intramolecular hydrogen bonding between the carbazole NH proton and the $\mathbf{C}=\mathbf{N}$ moiety included in the functional group was confirmed. Compared with the conjugated polymer P0, the conjugated polymers $\mathrm{P} 1$ and $\mathrm{P} 3$ had the absorption maxima at the longer wavelength region due to the intramolecular hydrogen bonding. The redshift of the ultraviolet-visible spectrum of the conjugated polymer P4 can be attributed to the donor(carbazole)-acceptor(pyridine) interaction. The conjugated polymers P1 and P3 showed similar photoluminescence spectra, and the fluorescence quantum yields were quite low. Although the conjugated polymer complex P3-Cu with the coordinated copper(II) ion was non-fluorescent, the conjugated polymer complex P3-Zn with the zinc(II) ion produced the longer wavelength emission than the undoped polymer P3.

Polymer Journal (2013) 45, 396-400; doi:10.1038/pj.2012.153; published online 8 August 2012

Keywords: carbazole; conjugated polymer; fluorene; intramolecular hydrogen bonding; metal ion

\section{INTRODUCTION}

Conjugated polymers have been studied extensively for use in various electronic and optoelectronic devices such as electroluminescence displays, photovoltaic cells and field-effect transistors. Many aromatic units, such as benzene, thiophene, pyridine, fluorene and carbazole, have been introduced in the conjugated polymer backbone. Among them, carbazole has many fascinating characteristics, as exemplified by the electrophilic substitution in the nitrogen atom and the aromatic ring, which produces diverse analogs and (electro)chemical and environmental stabilities. For instance, poly( $N$-vinylcarbazole)s have been used in xerographic applications as good photoconductors and hole-transporting materials. ${ }^{1}$ Owing to the high electron density at the 3,6-positions, the halogenation preferentially affords the 3,6-disubstituted derivatives, which enable the synthesis of poly(carbazole-3,6-diyl)s and copolymers. ${ }^{2}$ Moreover, Leclerc et al. developed the efficient preparation route of 2,7-dihalogenated carbazoles, and they revealed that poly(carbazole-2,7-diyl)s have wider pi-conjugation system than polymers with the 3,6-connection due to the nitrogen-bridged poly( $p$-phenylene) skeleton. ${ }^{3,4}$ Since then, poly(carbazole-2,7-diyl)s and copolymers have been attracting much attention as reliable materials for optoelectronic applications. Other carbazole-based conjugated polymers with various connection patterns were also prepared by many research groups. ${ }^{5-8}$ Recently, we reported partially conjugated fluorescent polymers that contain the carbazole-(bi)fluorene-carbazole chromophore separated by the insulating $p$-xylylene spacer. The report demonstrated that both the number of para-connected benzene rings and the interaction between the carbazole nitrogen lone pair and the oligophenylene moiety definitely affected the optical properties of the materials. ${ }^{9-11}$

As part of our ongoing studies on the structure-properties relationship of carbazole-based conjugated polymers, we herein synthesized three conjugated polymers that had the 1,8-difunctionalized/9-unsubstituted carbazole unit. To the best of our knowledge, although the carbazole derivatives with the free $\mathrm{NH}$ group and metal complexes thereof were reported, ${ }^{12-14}$ the related conjugated polymers have not been described. If the carbazole $\mathrm{NH}$ proton ( $\mathrm{pKa}$ approximately 20 in dimethyl sulfoxide) forms an intramolecular 
hydrogen bonding with the functional group at the 1,8-positions, or if carbazole can be converted into the $N$-metallated species, the electronic character of the carbazole ring can be modified to influence the optoelectronic properties of conjugated polymers. Hence, the effect of intramolecular hydrogen bonding and metal ion doping on the absorption and emission spectra are discussed in this paper.

\section{EXPERIMENTAL PROCEDURE}

\section{Materials}

All reactions were performed in a dry nitrogen atmosphere. 1,8-Dibromo-9Hcarbazole was synthesized according to the literature, ${ }^{15}$ and all synthetic procedures and characterization of carbazole monomers are described in the Supplementary Materials. 9,9-Dihexylfluorene-2,7-diboronic acid was purchased from Sigma-Aldrich (St Louis, MO, USA).

\section{Instrumentation}

The ${ }^{1} \mathrm{H}$ and ${ }^{13} \mathrm{C}$ nuclear magnetic resonance (NMR) spectra were recorded on a Bruker Avance 200 FT-NMR spectrometer (Bruker Biospin, Billerica, MA, USA) using tetramethylsilane $(\delta, 0.00)$ and $\operatorname{CDCl}_{3}(\delta, 77.0)$ as the internal reference peaks, respectively. The infrared (IR) spectra were recorded on a Jasco FT-IR 460Plus spectrophotometer (Jasco, Tokyo, Japan) using the attenuated total reflection (ATR) method. The melting points were determined on a Yanaco MP-500D micro melting point apparatus (Yanaco, Kyoto, Japan). The elemental analyses were performed on a PerkinElmer PE2400II elemental analyzer (Perkin Elmer, Waltham, MA, USA). The gel permeation chromatographic analyses were performed on a Tosoh DP-8020 pump (Tosoh, Tokyo, Japan) using the tandem TSK Multipore $\mathrm{H}_{\mathrm{XL}}-\mathrm{M}$ columns (with tetrahydrofuran (THF) as an eluent, flow rate $=1.0 \mathrm{ml} \mathrm{min}^{-1}, 40^{\circ} \mathrm{C}$ ), which were equipped with a refractive index detector (RI-8012). The number-averaged molecular weight $(M \mathrm{n})$ and molecular weight distribution $(\mathrm{Mw} / \mathrm{Mn})$ were estimated on the basis of the calibration curve made from standard polystyrene samples. Ultraviolet-visible (UV-Vis) and photoluminescence (PL) spectra were collected using a Shimadzu UV-1650PC spectrophotometer (Shimadzu, Kyoto, Japan) and a Shimadzu RF-5300PC spectrofluorometer (Shimadzu), respectively. The fluorescence quantum yields in the solution were determined relative to quinine sulfate in $0.5 \mathrm{M} \mathrm{H}_{2} \mathrm{SO}_{4}$ solution, which has a quantum yield of 0.55 .

\section{Polymerization}

Imine-functionalized polymer (P1) (Typical procedure): to a THF (10 ml) solution of monomer $4(0.12 \mathrm{~g}, 0.20 \mathrm{mmol})$ and 9,9-dihexylfluorene-2,7diboronic acids $(0.08 \mathrm{~g}, 0.20 \mathrm{mmol}), \mathrm{Pd}\left(\mathrm{PPh}_{3}\right)_{4}(2.3 \mathrm{mg}, 2.0 \mu \mathrm{mol})$ and $2 \mathrm{M}$ $\mathrm{Na}_{2} \mathrm{CO}_{3}(2 \mathrm{ml})$ were added, and the mixture was heated to reflux for $48 \mathrm{~h}$. After the solution was neutralized with $1 \mathrm{M} \mathrm{HCl}$, an aqueous phase was extracted with $\mathrm{CHCl}_{3}$. The combined organic phase was washed with saturated $\mathrm{NH}_{4} \mathrm{Cl}$ solution and brine, and then it was dried over $\mathrm{MgSO}_{4}$. The polymer was purified by precipitating in hot $\mathrm{CH}_{3} \mathrm{OH}$ and subjected to Soxhlet extraction with acetone for $6 \mathrm{~h}$ to obtain an orange powder. The yield was $0.10 \mathrm{~g}(65 \%) . M \mathrm{n}=11100(\mathrm{Mw} / \mathrm{Mn}=1.6) .{ }^{1} \mathrm{H}-\mathrm{NMR}\left(\delta, \mathrm{CDCl}_{3}\right) 0.63-0.95$ (10H, Alkyl), 0.97-1.28 (12H, Alkyl), 1.95-2.45 (22H, Alkyl), 6.87 (4H, bs, Ar), 7.65-8.05 (8H, Ar), $8.64(2 \mathrm{H}, \mathrm{bs}, \mathrm{Ar}), 8.68(2 \mathrm{H}, \mathrm{bs}, \mathrm{N}=\mathrm{CH}), 12.64(1 \mathrm{H}, \mathrm{bs}$, NH). IR (ATR) 665, 817, 852, 875, 1012, 1144, 1198, 1264, 1377, 1462, 1587, $2854,2927,3019,3373$. Similarly, other conjugated polymers were prepared by the Suzuki coupling polymerization and characterized by gel permeation chromatographic, ${ }^{1} \mathrm{H}-\mathrm{NMR}$ spectra and IR spectra as follows:

1,3-Oxazoline-functionalized polymer $(\mathbf{P 2}): M \mathrm{n}=6800(\mathrm{Mw} / \mathrm{Mn}=1.4)$. ${ }^{1} \mathrm{H}-\mathrm{NMR}\left(\delta, \mathrm{CDCl}_{3}\right) \quad 0.60-0.95$ (10H, Alkyl), 0.97-1.32 (12H, Alkyl), 1.56 (12H, bs, Me), 1.95-2.35 (4H, Alkyl), $4.27\left(4 \mathrm{H}, \mathrm{bs}, \mathrm{OCH}_{2}\right), 7.62-7.94(6 \mathrm{H}$, Ar), 8.25 (2H, bs, Ar), 8.59 (2H, bs, Ar), 12.27 (1H, bs, NH). IR (ATR) 681, 722, 820, 878, 999, 1055, 1146, 1194, 1262, 1364, 1464, 1602, 1646, 2856, 2929, 2957, 3022, 3326.

Pyridine-functionalized 9- $\mathrm{H}$ polymer $(\mathbf{P 3}): \mathrm{Mn}=6700(\mathrm{Mw} / \mathrm{Mn}=1.3) .{ }^{1} \mathrm{H}$ NMR $\left(\delta, \mathrm{CDCl}_{3}\right)$ 0.53-1.01 (10H, Alkyl), 1.01-1.35 (12H, Alkyl), 1.95-2.37 (4H, Alkyl), 7.22-7.40 (2H, Ar), 7.61-8.48 (12H, Ar), 8.61 (2H, bs, Ar), 8.97 (2H, bs, Ar), 13.33 (1H, bs, NH). IR (ATR) 738, 788, 820, 872, 1140, 1203, 1260, 1416, 1456, 1476, 1588, 2853, 2926, 3359.

Pyridine-functionalized 9-Me polymer (P4): $M n=5100(M w / M n=1.5)$. ${ }^{1} \mathrm{H}-\mathrm{NMR}\left(\delta, \mathrm{CDCl}_{3}\right)$ 0.57-0.92 (10H, Alkyl), 0.94-1.25 (12H, Alkyl), 1.94-2.30 (4H, Alkyl), 2.99 (3H, bs, Me), 7.22-7.40 (2H, Ar), 7.63-8.04 (12H, Ar), 8.58 (2H, bs, Ar), 8.80 (2H, bs, Ar). IR (ATR) 683, 747, 789, 820, 874, 1061, 1128 , $1186,1260,1402,1429,1454,1562,1584,2852,2926,3050$.

Phenyl-functionalized polymer (P0): $M \mathrm{n}=8400(\mathrm{Mw} / \mathrm{Mn}=1.6) .{ }^{1} \mathrm{H}-\mathrm{NMR}$ $\left(\delta, \mathrm{CDCl}_{3}\right)$ 0.60-0.95 (10H, Alkyl), 0.95-1.30 (12H, Alkyl), 1.95-2.33 (4H, Alkyl), 7.36-8.00 (18H, Ar), 8.51 ( $2 \mathrm{H}, \mathrm{bs}, \mathrm{Ar}), 8.58(1 \mathrm{H}, \mathrm{bs}, \mathrm{NH})$. IR (ATR) $701,770,818,871,1028,1156,1261,1415,1461,1496,1599,2851,2952$, $3028,3463$.

\section{RESULTS AND DISCUSSION}

\section{Polymer synthesis}

Three carbazole monomers that had the imine (4), 1,3-oxazoline (6), and pyridine (8) functional group at the 1,8-positions were synthesized from 1,8-dibromocarbazole. As the $\mathrm{N}$-methyl analog of monomer 8, monomer 9 was prepared to investigate the relationship between the intramolecular hydrogen bonding and the optical properties of the conjugated polymer. Monomer 11, which bore the phenyl group, was also synthesized. The monomer synthetic procedure is described in the Supplementary Materials. These carbazole monomers were subjected to the Suzuki coupling polymerization with 9,9-dihexylfluorene-2,7-diboronic acid (Scheme 1). In preparing
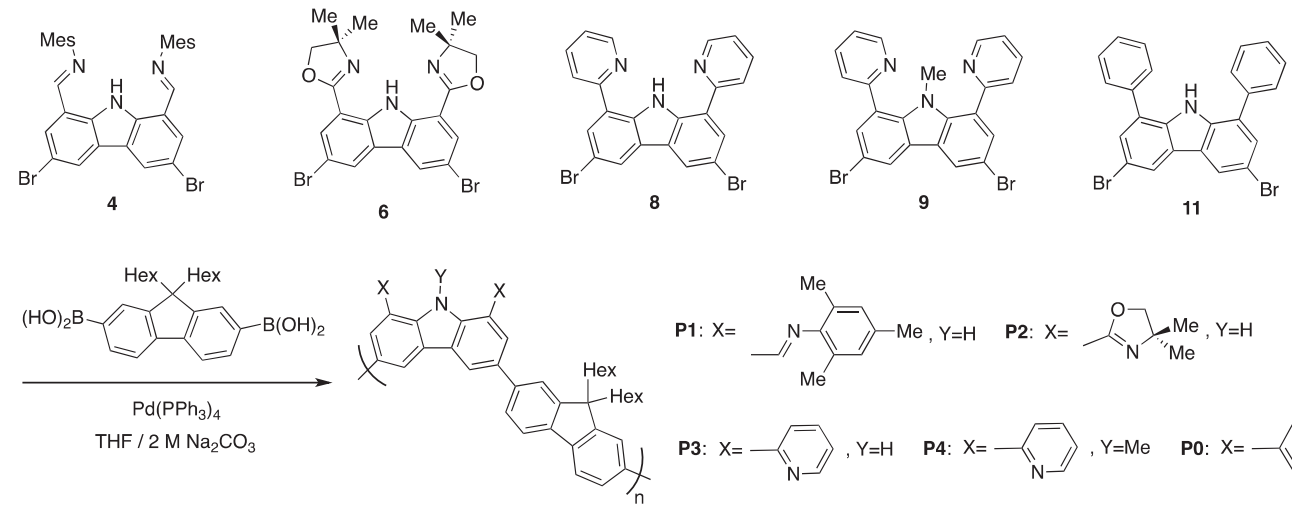

P1: $\mathrm{X}=$<smiles>CC=Nc1c(C)cc(N=[V])cc1C</smiles><smiles>[Y]c1ccc(C=[Y])cn1</smiles>

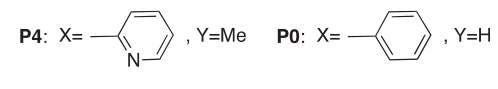

Scheme 1 Synthesis of conjugated polymers (P1-P4 and P0) using Suzuki coupling polymerization. 


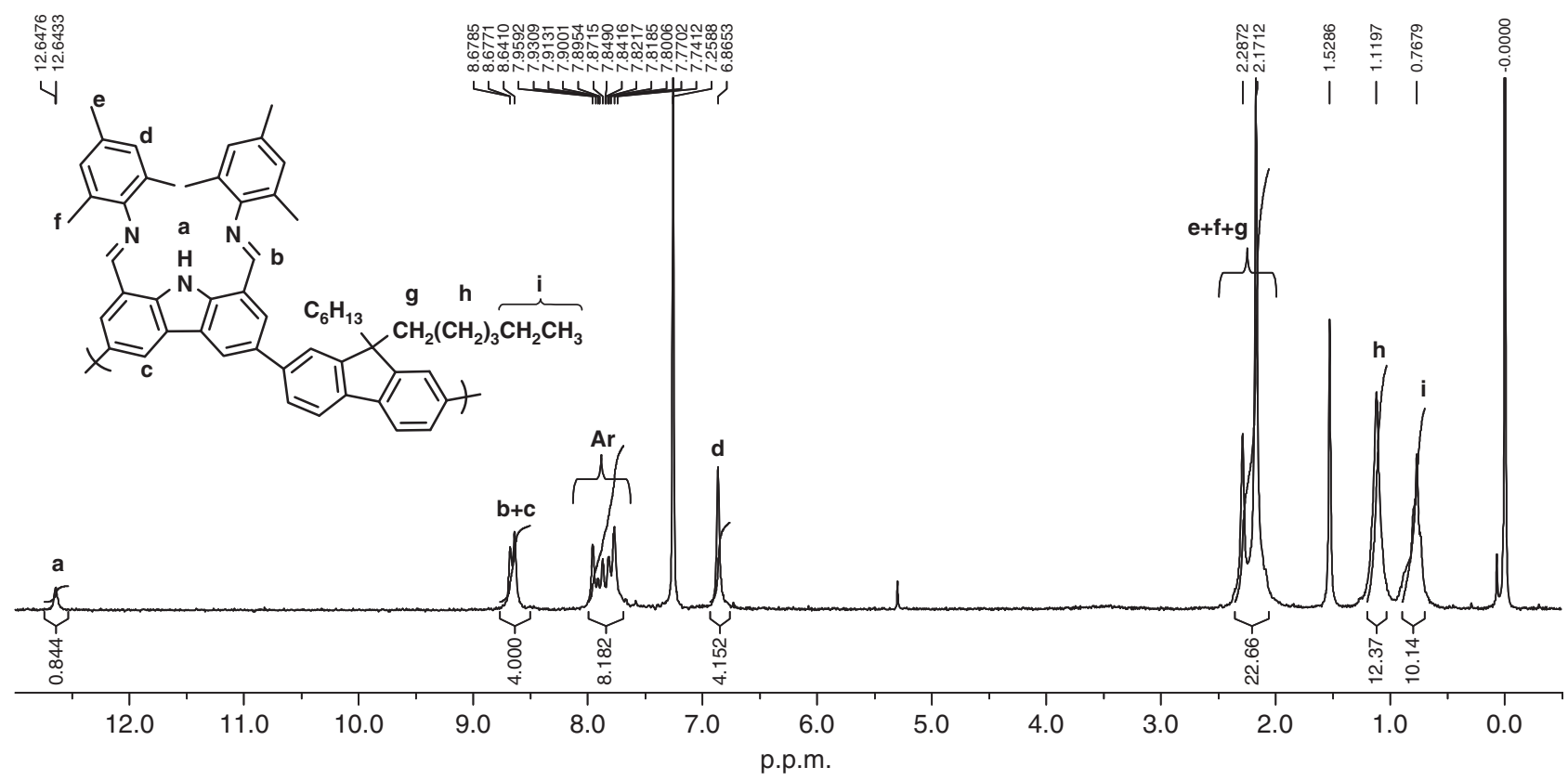

Figure $1{ }^{1} \mathrm{H}-\mathrm{NMR}$ spectrum of conjugated polymer $\mathbf{P} 1$ in $\mathrm{CDCl}_{3}(200 \mathrm{MHz})$.

the imine-functionalized conjugated polymer, we initially prepared 3,6-dibromo-1,8-bis((phenylimino)methyl)carbazole as the monomer and performed the Suzuki coupling polymerization. However, the imine $\mathrm{C}=\mathrm{N}$ bond was partially hydrolyzed to produce the formyl group, as it was confirmed by the ${ }^{1} \mathrm{H}-\mathrm{NMR}$ spectra, where the remaining imine functional group was calculated to be $80 \%$ from the integral ratio. Meanwhile, the polymerization that used monomer 4 with the sterically demanding imine functional group did not undergo hydrolysis to give the corresponding polymer P1, and the signal assignable to the imine proton was observed at 8.68 p.p.m. in the theoretical integral ratio (Figure 1). Similarly, other conjugated polymers were prepared and purified by precipitation in hot $\mathrm{CH}_{3} \mathrm{OH}$, followed by Soxhlet extraction with acetone. All conjugated polymers had good solubility in THF, $\mathrm{CHCl}_{3}$ and toluene. The numberaveraged molecular weight of the polymers ranged from 5100 to 11 100. The conjugated polymers P1-P3 showed the NH proton signal at $12-13$ p.p.m., which was shifted considerably to the downfield region than that of the conjugated polymer P0 (8.58 p.p.m.). The stretching $\mathrm{N}-\mathrm{H}$ vibration signal of the conjugated polymers P1-P3 were observed below $3400 \mathrm{~cm}^{-1}$, while that of conjugated polymer P0 was observed below $3463 \mathrm{~cm}^{-1}$. These results indicate that intramolecular hydrogen bonding occurred between the carbazole $\mathrm{NH}$ proton and the $\mathrm{C}=\mathrm{N}$ moiety that were included in the functional group at the 1,8-positions.

\section{Optical properties}

The UV-Vis and PL spectra of conjugated polymers P1-P4 were measured in THF solution (Figure 2), and the maximum wavelengths are listed in Table 1. In comparison with the conjugated polymer P0 $\left(\lambda_{\max }=340 \mathrm{~nm}\right.$, the UV-Vis and PL spectra are only given in the Supplementary Materials), which bore the phenyl group at the 1,8positions, the conjugated polymers P1-P3 showed the absorption maxima at the longer wavelength region. More specifically, the conjugated polymers P1 and P3 had absorption maxima $>360 \mathrm{~nm}$. Meanwhile, the conjugated polymer $\mathbf{P} 4$, which cannot form intramolecular hydrogen bonding, also showed a slightly red-shifted UV-
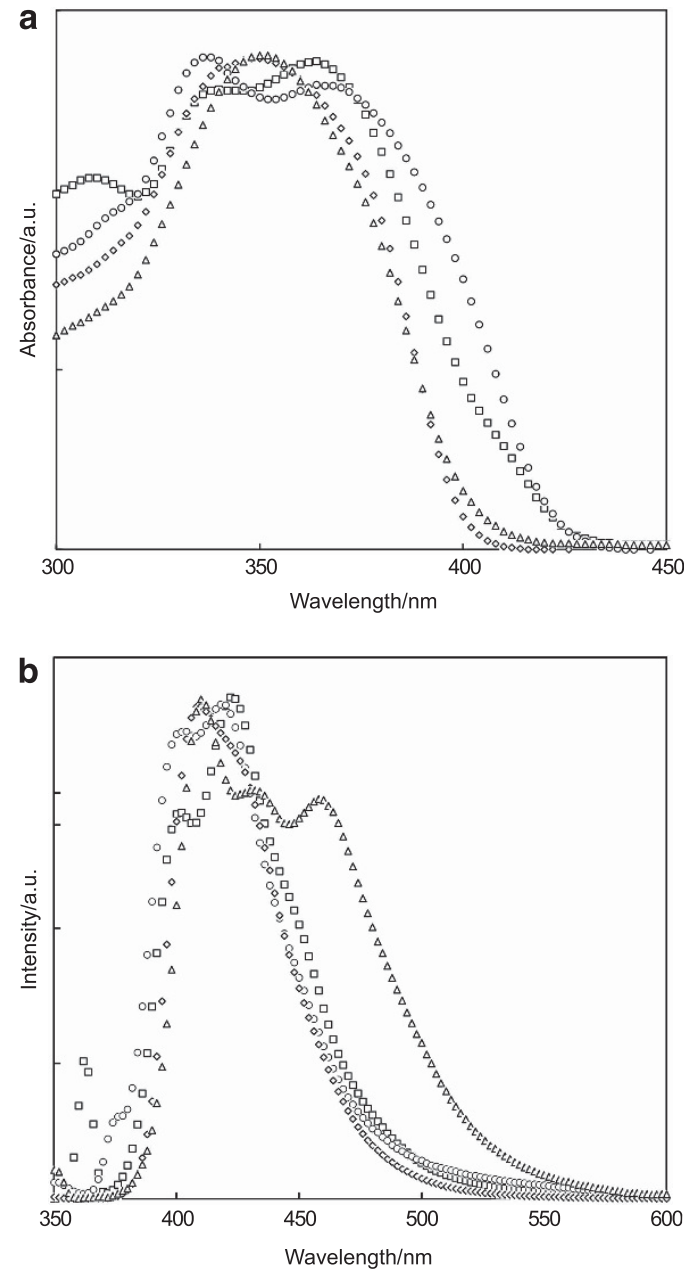

Figure 2 (a) UV-Vis and (b) PL spectra of the polymers in the THF solution $\left(10^{-5} \mathrm{M}\right)$ (circle: P1, triangle: P2, square: P3 and diamond: P4). 
Table 1 Optical properties of conjugated polymers in THF

\begin{tabular}{lccr}
\hline & & \multicolumn{2}{c}{$P L$} \\
\cline { 2 - 4 } & $\lambda_{\max } / n m$ & $\lambda_{\max } / n m$ & \multicolumn{1}{c}{$Q Y^{\mathrm{a}}$} \\
\hline P1 & $365^{\mathrm{b}}$ & 401,418 & $<0.01$ \\
P2 & 351 & $410,431,458$ & 0.02 \\
P3 & $363^{\mathrm{b}}$ & 402,423 & $<0.01$ \\
P4 & 349 & 410,418 & 0.19
\end{tabular}

Abbreviations: PL, photoluminescence; UV-Vis, Ultraviolet-visible.

${ }^{a} Q u a n t u m$ yields relative to quinine sulfate as standard $(0.55)$.

bonly absorption maxima at the longer wavelength region are described.
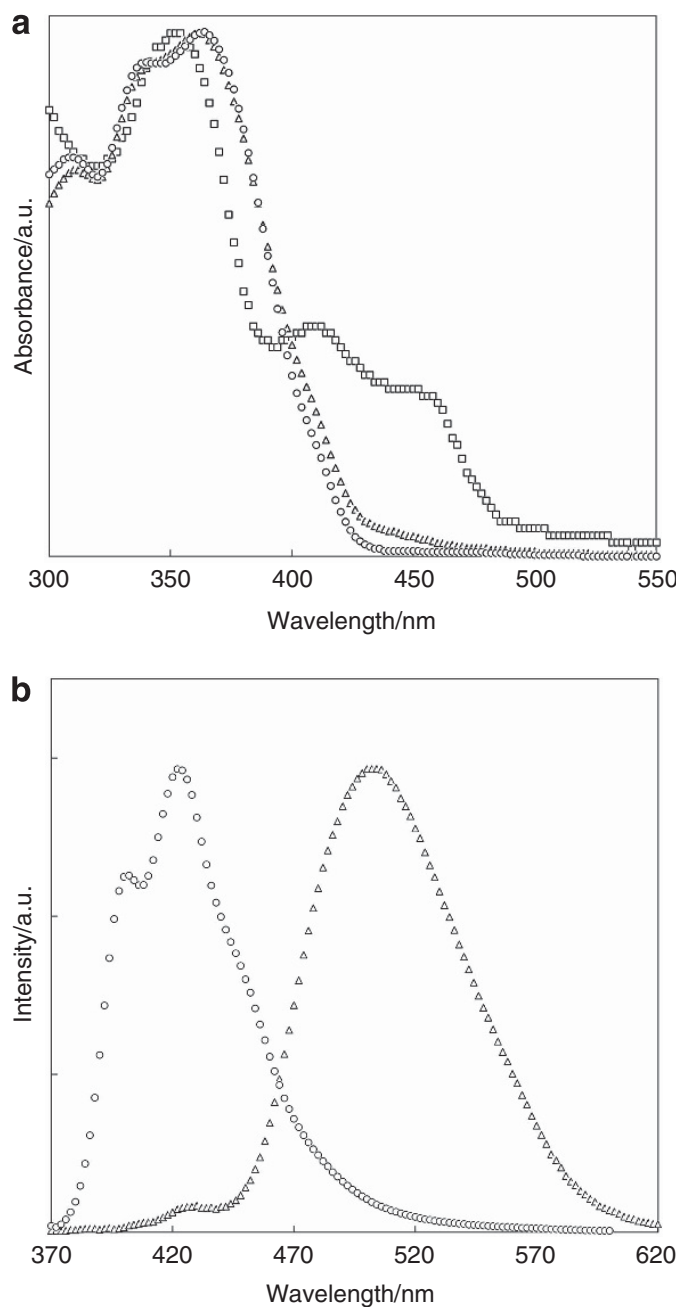

Figure 3 (a) UV-Vis and (b) PL spectra of the polymers in the THF solution $\left(10^{-5} \mathrm{M}\right.$ ) (circle: P3, triangle: P3-Zn and square: P3-Cu).

Vis spectra (and the onset wavelength), and the absorption maximum was observed at $349 \mathrm{~nm}$. This result can be explained by the electronic interaction between the donor (carbazole) and the acceptor (pyridine).$^{16}$ Therefore, the absorption maximum at $363 \mathrm{~nm}$ observed in the conjugated polymer $\mathbf{P 3}$ most likely originated from the intramolecular hydrogen bonding. All conjugated polymers emitted weak fluorescence, and the emission maximum wavelengths depended on the functional group at the 1,8-positions of the carbazole unit. The conjugated polymers $\mathbf{P} \mathbf{1}$ and $\mathbf{P 3}$, which can form strong intramolecular hydrogen bonding (vide supra), showed similar PL spectra, and the fluorescence quantum yields were quite low $(\mathrm{QY}<0.01)$. As previously reported for 7 - $\left(2^{\prime}\right.$-pyridyl)indole, ${ }^{17}$ the excited state intramolecular proton transfer might quench the fluorescence emission from these conjugated polymers. In contrast, the conjugated polymer $\mathbf{P 4}$, which cannot form intramolecular hydrogen bonding, emitted visible blue light with peak maxima at 410 and $418 \mathrm{~nm}$. Because the conjugated polymer P0 emitted purple light $\left(\lambda_{\max }=399 \mathrm{~nm}\right)$, the conjugated polymer $\mathbf{P} 4$ was proven to have the wider pi-conjugated system due to the donor-acceptor interaction as previously described. The conjugated polymer P2 showed the broad PL spectrum with three peak maxima at 410,431 and $458 \mathrm{~nm}$. Again, the fluorescence quantum yield was low $(\mathrm{QY}=0.02)$ because of the intramolecular hydrogen bonding.

Subsequently, the influence of the metal ion doping was investigated using the conjugated polymer $\mathbf{P} 3$, which bore the pyridine functional group. After the carbazole $\mathrm{NH}$ proton at the 9-position was abstracted with $\mathrm{NaH}$, an equimolar amount of $\mathrm{ZnCl}_{2}$ or $\mathrm{CuCl}_{2}$ was added to measure the UV-Vis and PL spectra in the THF solution (Figure 3). The conjugated polymer complex P3-Zn with the coordinated zinc(II) ion had a similar UV-Vis spectrum to the undoped polymer P3. The absorption maximum wavelength was $360 \mathrm{~nm}$, and a small tailing to the longer wavelength region was observed. The conjugated polymer complex P3-Cu with a coordinated copper(II) ion showed new absorption peaks at 406 and $450 \mathrm{~nm}$, which may be assigned to the metal-to-ligand charge transfer transition. Although the conjugated polymer complex P3-Cu was non-fluorescent, the conjugated polymer complex P3-Zn emitted a green light with a maximum wavelength at $500 \mathrm{~nm}$. The fluorescence quantum yield was approximately 0.02 , which was larger than that of the undoped polymer P3 $(<0.01)$. Thus, the inhibition of the intramolecular hydrogen bonding and the rigidification by the complex formation with the zinc(II) ion resulted in the redshift and the fluorescence enhancement of the conjugated polymer complex P3-Zn.

\section{CONCLUSIONS}

Three conjugated polymers with alternating carbazole and fluorene units were synthesized using the Suzuki coupling polymerization, where the 1,8-positions of the carbazole were functionalized with imine, 1,3-oxazoline and pyridine. The intramolecular hydrogen bonding between the carbazole $\mathrm{NH}$ proton and the $\mathrm{C}=\mathrm{N}$ moiety included in the functional group was confirmed by the ${ }^{1} \mathrm{H}-\mathrm{NMR}$ and IR spectra. Compared with the polymer bearing the phenyl group at the 1,8-positions, all polymers showed the redshift of the UV-Vis spectra, which could be explained by the intramolecular hydrogen bonding and/or the donor-acceptor interaction. The formation of an intramolecular hydrogen bonding decreased the fluorescence quantum yields of the conjugated polymer. Metal ion doping had a large impact on the PL spectra, where the coordination of copper(II) ion completely quenched the fluorescence emission, but the coordination of zinc(II) ion resulted in an increase and a redshift of the PL spectra.

\section{CONFLICT OF INTEREST}

The authors declare no conflict of interest. 
1 Grazulevicius, J. V., Strohriegl, P., Pielichowski, J. \& Pielichowski, K. Carbazolecontaining polymers: synthesis, properties and applications. Prog. Polym. Sci. 28, 1297-1353 (2003)

2 Morin, J. -F., Leclerc, M., Aèds, D. \& Siove, A. Polycarbazoles: 25 years of progress. Macromol. Rapid Commun. 26, 761-778 (2005).

3 Blouin, N. \& Leclerc, M. Poly(2,7-carbazole)s: structure-property relationships. Acc. Chem. Res. 41, 1110-1119 (2008).

4 Boudreault, P. -L., Blouin, N. \& Leclerc, M. Poly(2,7-carbazole)s and related polymers. Adv. Polym. Sci. 212, 99-124 (2008).

5 Wu, C. -W. \& Lin, H. -C. Synthesis and characterization of kinked and hyperbranched carbazole/fluorene-based copolymers. Macromolecules 39, 7232-7240 (2006).

6 Liu, R., Xiong, Y., Zeng, W., Wu, Z., Du, B., Yang, W., Sun, M. \& Cao, Y. Extremely colorstable blue light-emitting polymers based on alternating 2,7-fluorene-co-3,9-carbazole copolymer. Macromol. Chem. Phys. 208, 1503-1509 (2007).

7 Tamura, K., Shiotsuki, M., Kobayashi, N., Masuda, T. \& Sanda, F. Synthesis and properties of conjugated polymers containing 3,9- and 2,9-linked carbazole units in the main chain. J. Polym. Sci. Part A Polym. Chem. 47, 3509-3517 (2009).

8 Michinobu, T., Osako, H. \& Shigehara, K. Synthesis and properties of conjugated poly(1,8-carbazole)s. Macromolecules 42, 8172-8180 (2009).

9 Takagi, K., Nakagawa, T. \& Takao, H. Synthesis of carbazole-based light emitting polymers incorporating 2,5-bis(phenylethenyl)-4-decyloxyanisole as collateral fluorophore. J. Polym. Sci. Part A Polym. Chem. 46, 8141-8148 (2008).
10 Takagi, K., Nakagawa, T. \& Takao, H. Synthesis and light-emitting properties of carbazole-based copolymers bearing cyano-substituted arylenevinylene chromophore. J. Polym. Sci. Part A Polym. Chem. 48, 91-98 (2010).

11 Takagi, K., Takao, H. \& Nakagawa, T. Synthesis and characterization of nitrogen-linked carbazole-containing fluorescent polymers. J. Polym. Sci. Part A Polym. Chem 48, 3729-3735 (2010).

12 Britovsek, G. J. P., Gibson, V. C., Hoarau, O. D., Spitzmesser, S. K., White, A. J. P. \& Williams, D. J. Iron and cobalt ethylene polymerization catalysts: variations on the central donor. Inorg. Chem. 42, 3454-3465 (2003).

13 Inoue, M., Suzuki, T. \& Nakada, M. Asymmetric catalysis of nozaki-hiyama allylation and methallylation with a new tridentate bis(oxazolinyl)carbazole ligand. J. Am. Chem. Soc. 125, 1140-1141 (2003)

14 Mudadu, M. S., Singh, A. N. \& Thummel, R. P. Preparation and study of 1,8-Di(pyrid$2^{\prime}$-yl)carbazoles. J. Org. Chem. 73, 6513-6520 (2008).

15 Suzuki, T., Kinoshita, A., Kawada, H. \& Nakada, M. A. New asymmetric tridentate carbazole ligand: its preparation and application to nozaki-hiyama allylation. Synlet 4 570-572 (2003)

16 Michinobu, T., Osako, H., Seo, C., Murata, K., Mori, T. \& Shigehara, K. Multicolor emission and thin film transistor properties of 1,8-diethynylcarbazole- based conjugated copolymers. Polymer 52, 5756-5763 (2011).

17 Wiosna, G., Petkova, I., Mudadu, M. S., Thummel, R. P. \& Waluk, J. Intra- and intermolecular fluorescence quenching in 7-(pyridyl)indoles. Chem. Phys. Lett. 400, 379-383 (2004).

Supplementary Information accompanies the paper on Polymer Journal website (http://www.nature.com/pj) 